Reprod. Nutr. Dévelop., 1987, 27 (1 B), 263-264.

\title{
Variabilité de la dégradation dans le rumen " in sacco " des constituants des fourrages verts
}

P. LE GOFFE, C. MESNIL, R. VÉRITÉ

Station de Recherches sur la Vache laitière, I.N.R.A., Saint-Gilles, 35590 L'Hermitage, France.

Summary. The rumen degradation pattern of green forage components in nylon bags varies considerably due to differences between cows and days. The rate of disappearance of dry matter in a control sample after $8 \mathrm{~h}$ reduced within-forage variability by cancelling out the differences between cows.

L'utilisation des sachets de nylon pour comparer la dégradation des aliments dans le rumen exige de normaliser les conditions de mesure. Même si un effort de standardisation (technique, régime) est fait, il subsiste des variations dans l'activité de la population microbienne et l'exécution de la mesure (Setala, 1983 ; Mehrez et Orskov, 1977). Dans cette étude, réalisée sur des fourrages verts, nous avons essayé de : 1) quantifier certaines de ces variations qui se manifestent par des différences entre vaches et jours, 2) les prendre en compte à l'aide d'un témoin.

Matériel et méthodes. 24 fourrages verts, jeunes pour la plupart, congelés dès la récolte puis lyophilisés, ont servi à cette étude avec un témoin luzerne déshydratée. Pour chacun, les taux de disparition (TD) de la matière sèche (MS) et de l'azote $(\mathrm{N})$ ont été mesurés in sacco après $2,4,8,16$ et $48 \mathrm{~h}$ de séjour dans le rumen, avec 6 répétitions ( 2 vaches $\times 3$ jours). En pratique, les fourrages ont été testés en 6 séries de 4 (plus le témoin), comportant 3 jours de mesures et 25 sachets/vache/jour. Chaque sachet (pore $50 \mu$, dimensions internes $6 \times 11 \mathrm{~cm}$ ) a été rempli avec $3 \mathrm{~g}$ de fourrage ou de témoin broyés à la grille de $0,8 \mathrm{~mm}$. Les 2 vaches fistulées du rumen, taries et gestantes, ont reçu $8 \mathrm{~kg}$ de foin de trèfle violet par jour en 2 fractions égales à $8 \mathrm{~h}$ et $17 \mathrm{~h}$. Pour chaque aliment et chaque temps de séjour, l'azote des résidus de sachets a été dosé sur l'échantillon moyen de chaque vache (regroupement des 3 jours) sauf à 48 h (échantillon moyen de 2 vaches). Les dégradations théoriques de la matière sèche et de l'azote de chaque aliment ont été calculées à partir des cinétiques de disparition en admettant un taux de passage des particules de $5 \%$ par heure (calcul pas à pas).

Résultats et discussion. La précision de la mesure du TD-MS, appréciée par la variabilité (écart-type s) entre les témoins, est meilleure à la fin de l'incubation ( $s$ de $1 \%$ à $48 \mathrm{~h}$ ) qu'au début (s entre 2,9 et 3,9\% jusqu'à $16 \mathrm{~h}$ ); ceci est encore plus prononcé pour l'écart-type intra-fourrage (tabl. 1) et provient de différences très significatives existant entre jours et surtout entre vaches à 2, 4 et $8 \mathrm{~h}$.

Toutefois, les variations intra-fourrage du TD-MS à 2, 4 ou $8 \mathrm{~h}$ ont été très liées à celles du TD-MS du témoin de même durée $(2,4$ ou $8 \mathrm{~h})$ ou, quoique moins étroitement, à celles du seul TD-MS du témoin à $8 \mathrm{~h}$. Ce dernier a été retenu comme unique covariable pour corriger les valeurs du TD-MS des fourrages et les ramener ainsi à la valeur moyenne du témoin à $8 \mathrm{~h}$. La correction diminue l'écart-type intra-fourrage qui reste cependant supérieur d'environ $1 \%$ 
(tabl. 1) à l'écart-type résiduel après prise en compte des effets vache et jour. Elle réduit les effets jour, l'interaction vache $\times$ jour et surtout l'effet vache qui disparaît à $2 \mathrm{~h}$ et $4 \mathrm{~h}$.

TABL. 1. - Variabilité du taux de disparition in sacco et de la dégradation théorique de la matière sèche de fourrages verts avant ou après correction par un témoin (1).

\begin{tabular}{|c|c|c|c|c|c|c|}
\hline p. 100 & \multicolumn{5}{|c|}{ Taux de disparition au bout de } & $\begin{array}{l}\text { Dégradation } \\
\text { théorique }\end{array}$ \\
\hline $\begin{array}{l}\text { Moyenne } \ldots \ldots \ldots \ldots \\
s \text { intra-fourrage } \ldots \ldots \ldots \\
\text { s résiduel } \ldots \ldots \ldots \ldots\end{array}$ & $\begin{array}{r}57,1 \\
3,3 \\
2,0\end{array}$ & $\begin{array}{r}64,2 \\
4,6 \\
2,5\end{array}$ & $\begin{array}{r}74,9 \\
4,6 \\
1,8\end{array}$ & $\begin{array}{r}85,4 \\
1,7 \\
1,2\end{array}$ & $\begin{array}{r}91,4 \\
0,5 \\
0,4\end{array}$ & $\begin{array}{r}79,6 \\
1,7 \\
0,8\end{array}$ \\
\hline 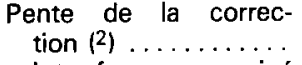 & $0,40 \pm 0,12$ & $0,67 \pm 0,15$ & $0,84 \pm 0,10$ & $0,02 \pm 0,06$ & $0,03 \pm 0,02$ & $0,27 \pm 0,04$ \\
\hline
\end{tabular}

(1) Correction par le taux de disparition de la matière sèche du témoin au bout de $8 \mathrm{~h}$.

(2) Pente et écart-type de la régression fourrage/témoin $8 \mathrm{~h}$.

Pour l'azote (TD moyens 68,$5 ; 76,2 ; 86,4 ; 92,8$ et $94,4 \%$ à $2,4,8,16$ et $48 \mathrm{~h}$ ), la meilleure correction des TD mesurés à $2,4,8 \mathrm{~h}$ est obtenue en prenant comme covariable le TD-MS (et non d'azote) du témoin à $8 \mathrm{~h}$. L'effet vache disparaît alors totalement.

Les écarts types intra-fourrage des dégradations théoriques (valeurs moyennes 79,6 et $86,4 \%$ pour la MS et l'N respectivement) calculées à partir des TD bruts sont notables (en particulier différences très significatives entre vaches), quoique plus faibles que ceux observés pour ces derniers en début d'incubation. Le calcul des dégradabilités théoriques à partir des TD corrigés réduit cet écarttype intra-fourrage et plus encore l'effet animal (différences entre vaches qui passent de 2,2 à $0,6 \%$ et de 1,9 à $0,5 \%$ pour la $M S$ et I'N respectivement). L'ajustement direct des dégradations théoriques sur le TD-MS du témoin à $8 \mathrm{~h}$ (tabl. 1) permet de retrouver les mêmes valeurs corrigées.

L'usage d'un témoin devrait permettre de réduire le nombre de répétitions et/ou de mieux discriminer les aliments et de les comparer mêrmes s'ils ont été testés sur des animaux ou à des périodes différentes. On peut peut-être envisager également une harmonisation des résultats obtenus en des lieux différents ou dans d'autres conditions différentes (régimes, niveau d'alimentation...).

Mehrez A. Z., Orskov E. R., 1977. J. agric. Sci., Camb., 88, 645-650.

Setala J., 1983. J. Sci. Agric. Soc. Finland., 55, 1-78. 